\title{
77 PREVALENCE OF SECONDARY IMMUNOTHERAPEUTIC TARGETS IN THE ABSENCE OF ESTABLISHED IMMUNE BIOMARKERS IN SOLID TUMORS
}

Paul DePietro*, Mary Nesline, Yong Hee Lee, RJ Seager, Erik Van Roey, Shuang Gao, Vincent Giamo, Blake Burgher, Sean Glenn, Shengle Zhang, Roger Klein, Sarabjot Pabla, Jeffrey Conroy. OmnSeq, Inc., Buffalo, NY, USA

Background Immune checkpoint inhibitor-based therapies have achieved impressive success in the treatment of several cancer types. Predictive immune biomarkers, including PD-L1, MSI and TMB are well established as surrogate markers for immune evasion and tumor-specific neoantigens across many tumors. Positive detection across cancer types varies, but overall $\sim 50 \%$ of patients test negative for these primary immune markers. ${ }^{1}$ In this study, we investigated the prevalence of secondary immune biomarkers outside of PD-L1, TMB and MSI. Methods Comprehensive genomic and immune profiling, including PD-L1 IHC, TMB, MSI and gene expression of 395 immune related genes was performed on 6078 FFPE tumors representing 34 cancer types, predominantly composed of lung cancer $(36.7 \%)$, colorectal cancer $(11.9 \%)$ and breast cancer (8.5\%). Expression levels by RNA-seq of 36 genes targeted by immunotherapies in solid tumor clinical trials, identified as secondary immune biomarkers, were ranked against a reference population. Genes with a rank value $\geq 75$ th percentile were considered high and values were associated with PD-L1 (positive $\geq 1 \%$ ), MSI (MSI-H or MSS) and TMB (high $\geq 10$ $\mathrm{Mut} / \mathrm{Mb}$ ) status. Additionally, secondary immune biomarker status was segmented by tumor type and cancer immune cycle roles.

Results In total, $41.0 \%$ of cases were PD-L1+, 6.4\% TMB+, and $0.1 \%$ MSI-H. $12.6 \%$ of cases were positive for $>2$ of these markers while $39.9 \%$ were triple negative (PD-L1-/ TMB-/MSS). Of the PD-L1-/TMB-/MSS cases, 89.1\% were high for at least one secondary immune biomarker, with $69.3 \%$ having $\geq 3$ markers. PD-L1-/TMB-/MSS tumor types with $\geq 50 \%$ prevalence of high secondary immune biomarkers included brain, prostate, kidney, sarcoma, gallbladder, breast, colorectal, and liver cancer. High expression of cancer testis antigen secondary immune biomarkers (e.g., NY-ESO-1, LAGE1A, MAGE-A4) was most commonly observed in bladder, ovarian, sarcoma, liver, and prostate cancer $(\geq 15 \%)$. Tumors demonstrating T-cell priming (e.g., CD40, OX40, CD137), trafficking (e.g., TGFB1, TLR9, TNF) and/or recognition (e.g., CTLA4, LAG3, TIGIT) secondary immune biomarkers were most represented by kidney, gallbladder, and sarcoma $(\geq 40 \%)$, with melanoma, esophageal, head \& neck, cervical, stomach, and lung cancer least represented $(\geq 15 \%)$.

Conclusions Our studies show comprehensive tumor profiling that includes gene expression can detect secondary immune biomarkers targeted by investigational therapies in $\sim 90 \%$ of PD-L1-/TMB-/MSS cases. While genomic profiling could also provide therapeutic choices for a percentage of these patients, detection of secondary immune biomarkers by RNA-seq provides additional options for patients without a clear therapeutic path as determined by PD-L1 testing and genomic profiling alone.

\section{REFERENCE}

1. Huang R S P, Haberberger J, Severson E, et al. A pan-cancer analysis of PD-L1 immunohistochemistry and gene amplification, tumor mutation burden and microsatellite instability in 48,782 cases. Mod Pathol 2021;34: 252-263. 